\title{
Nonlinear Wave Loads on High-rise Pile Cap Structures in the Donghai Bridge Wind Farm
}

\author{
Ling Chen \\ School of Engineering Sciences, University of Chinese Academy of Sciences \\ Beijing, China
}

Jifu Zhou, Xu Wang and Zhan Wang

Key Laboratory for Mechanics in Fluid Solid Coupling Systems, Institute of Mechanics

Chinese Academy of Sciences, Beijing, China

\begin{abstract}
A new type of bottom-fixed structure, the so-called high-rise pile cap foundation, has been proposed and used to support offshore wind turbines in the Donghai Bridge Wind Farm, China. Engineers are unaware of the wave load mechanisms for this new structure. Using the Navier-Stokes equations and volume of fluid technique, a fully nonlinear numerical wave tank is established to investigate free surface wave loads and moments for the new structure. The interaction between the cap and piles are discussed in detail. In the case of fully nonlinear waves, the maximum horizontal wave load on all the piles with the cap can increase by $30 \%$ compared with those without the cap, and the maximum horizontal wave load on a single pile is nearly doubled. The horizontal wave load on the cap with the piles can increase by about $15 \%$, while the vertical wave load decreases slightly. The conventional Morison formula and diffraction theory generally underestimate the wave loads on the piles and the cap as well.
\end{abstract}

\section{INTRODUCTION}

In 2010, China built the first large-scale offshore wind farm in Asia, the Donghai Bridge Wind Farm in the East China Sea. Unlike the monopole foundations widely used in the North Sea, the foundation for wind turbines in the Donghai Bridge Wind Farm is a high-rise pile cap structure. It was proposed for the following reasons. First, the hydrogeological conditions in the East China Sea are quite different from those in the North Sea. Multilayer soft clay with a total thickness of more than $25 \mathrm{~m}$ covers the seabed. For the safety and stability of offshore wind turbine structures on this soft clay, the sea bed near a large-diameter monopile or tripod foundation for large installation capacity should be protected by a wide range of surface hardening. But this will greatly impact marine ecosystems, which contrasts with environmental protection requirements (Chen et al., 2016). Second, severe typhoons frequently hit this area; thus more stringent requirements on the structure's strength and stability need to be met. Third, there is a busy fairway across the wind farm, making the cap necessary for preventing collisions between the foundation and ships.

The high-rise pile cap structure consists of a cap platform and eight piles. Two photos of this structure under construction and a sketch model can be seen in Fig. 1. The cap platform is the reinforced concrete structure with a 3-meter-high cylinder and an upper 1.5-meter-high frustum. The diameters of the cylinder and the top of frustum are $14 \mathrm{~m}$ and $11 \mathrm{~m}$, respectively. The cap bottom is a flat plate with girders on the inside. Eight inclined supporting piles below the cap are evenly distributed around the cap

Received August 27, 2017; updated and further revised manuscript received by the editors January 17,2018 . The original version (prior to the final updated and revised manuscript) was presented at the Twenty-seventh International Ocean and Polar Engineering Conference (ISOPE-2017), San Francisco, California, June 25-30, 2017.

KEY WORDS: Offshore wind turbine, high-rise pile cap foundation, wave loads, impact effect, Donghai Bridge Wind Farm. bottom, each with a diameter of $1.7 \mathrm{~m}$ and a 5.5:1 slope ratio. Other detailed information about this structure can be found in Lin et al. (2007), Lu (2010), and Chang et al. (2014). This new foundation has many advantages over other conventional supporting systems, such as high stiffness, manageable risk, economical cost, anticollision, environmental hospitality, etc. (Chen et al., 2016). As a result, it has served as an in-situ test model to support offshore wind turbines (Lin et al., 2007).

Although it symbolizes a benchmark project in China, or even in Asia, it still needs more verification of extreme events. One of the fundamental issues is the extreme wave loads on the highrise pile cap foundation. Because of the complexity of the structures and the strong nonlinearity of extreme waves, traditional methods, such as the Morison equation and the diffraction theory, are unable to estimate the wave loads accurately. Moreover, the cap just pierces the still water surface so that it is sometimes exposed to air and sometimes submerged, especially in the case

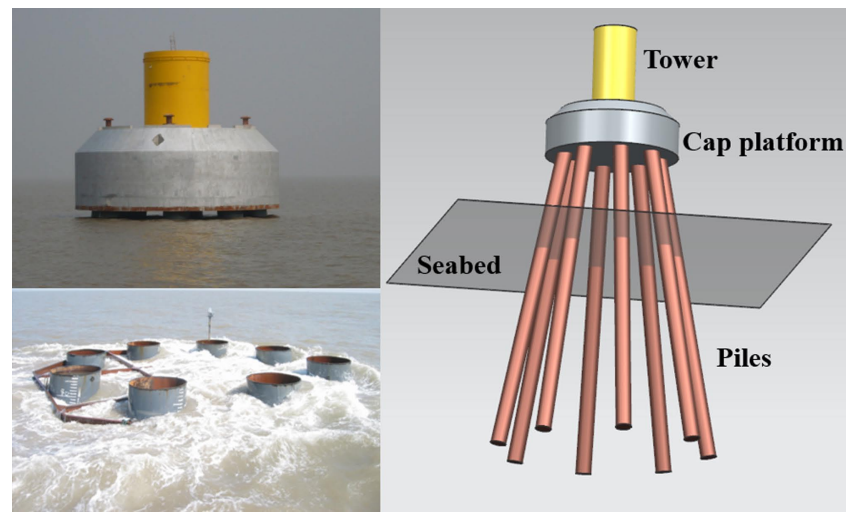

Fig. 1 Photos of a high-rise pile cap structure under construction in the Donghai Bridge Wind Farm (left) and the foundation sketch model (right) 
of large-amplitude waves. This phenomenon causes the free surface effect to be more pronounced, and it usually produces wave impact loads. In this project, a safety assessment of the foundation requires an estimation of wave loads acting on the structures. Even though the Morison equation can predict the wave force of a single pile or pile group well, it is not accurate enough to estimate that of the cap or the whole structure. Additionally, most standard engineering tools by now are not capable of accurately modelling them, and the design parameters are therefore often based on model tests.

Computational fluid dynamics (CFD) analyses of wave impacting on this new type of structures have been rarely undertaken. For the high-rise pile cap foundation, the wave loads include not only the usual wave force but also the load resulted from surface wave impacting the bottom of the cap. This impact-related load can play a significant role in estimating total wave loads. In this respect, similar work in wave-in-deck loading has been achieved. Bredmose and Jacobsen (2011) presented a numerical simulation of wave impacts on a monopile and then showed a subsequent vertical impact on the inspection platform. Schellin et al. (2011) demonstrated that a modern CFD technique was able to predict the loads on a typical jack-up platform; in particular, the wavein-deck load acting on the hull in freak waves was taken into account. Seiffert et al. (2014) investigated horizontal and vertical forces acting on a two-dimensional horizontal plate as a result of solitary waves by conducting a series of laboratory experiments as well as calculations using the InterFoam solver in OpenFOAM.

However, there is a difference between the wave-in-deck loads and the high-rise pile cap foundation loads. The investigations of the wave-in-deck loads are mostly on the direct vertical impact load of the horizontal plate. In the case of high-rise pile cap structures in this research, the cap bottom is similar to the deck and suffers from the vertical impact load, but the piles are not directly affected by the wave impact. They are subjected to the secondary influence of the high pressure resulting from the impact. So the traditional empirical formulas of the impact loads of horizontal plates cannot properly deal with this problem. In this situation, the most direct and effective method is to use the CFD technique to obtain the pile loads via the pressure field during impact.

As a CFD technique, the numerical simulation of nonlinear waves or extreme waves and their interaction with structures has been paid much attention. Many previous studies have been carried out on wave generation and absorption (Morgan et al., 2010; Jacobsen et al., 2012; Higuera et al., 2013) and the wave-structure interaction, including vertical cylinders (Chen et al., 2014; Paulsen et al., 2014a; Kamath et al., 2015; Hu et al., 2016; Lin et al., 2017) and the surface-piercing bodies, such as offshore floating bodies, coastal bridge decks, and so on (Stansberg et al., 2005; Li and Lin, 2012; Higuera et al., 2014; Seiffert et al., 2015). When waves interact with these structures around the free water surface, the impact effect cannot be ignored, although it can hardly be predicted by the diffraction theory. However, much research on impact loads via the air-water two-phase model has shown that numerical simulations can well describe the actual impact loads (Kleefsman et al., 2005; Iwanowski et al., 2009; Hayatdavoodi et al., 2014; Azadbakht and Yim, 2016). The geometry and position of the structures are the main factors in determining the magnitude of impact loads under certain wave climates. In engineering designs, the appropriate structural optimization can effectively reduce wave impact loads (Cuomo et al., 2009; Seiffert et al., 2015).

In the present investigation, the interaction between waves, piles, and the cap are analyzed by CFD computations. In the next section, the numerical model is presented and validated by testing the wave generation, wave force, and impact pressure field. Then, the test structures, physical domain, and wave parameters are introduced based on actual conditions; the grid convergence is also discussed. Afterward, the wave loads on the structure members are calculated, and the mutual influence of the cap and the piles is discussed. At the same time, the wave loads estimated by the conventional methods are compared with the numerical results. Finally, a summary and some conclusions are given. It should be noted that this paper focuses on the wave force of the high-rise pile cap structure. When discussing the wave loads of the cap and of the piles, the force transferred to the piles from the pile-cap intersection is not considered.

\section{NUMERICAL MODEL}

\section{Governing Equations}

To deal with fully nonlinear wave conditions, the Navier-Stokes equations are generally used to describe large deformations of the free surface and even wave breaking, especially near the structures. We use the incompressible Navier-Stokes solver interFoam (available in the OpenFOAM Toolbox), which is designed for simulating the evolution of the interface between two phases at constant temperature. The interface is captured through the volume of fluid (VOF) technique (Hirt and Nichols, 1981), which uses the volume fraction to define the volume portion of each phase occupying in the cell. A combined PISO-SIMPLE (PIMPLE) algorithm is adopted to solve the velocity-pressure coupling: it uses the Semi-Implicit Method for Pressure-Linked Equations (SIMPLE) to calculate the pressure iteratively from velocity on local mesh based on the Navier-Stokes equations, and it applies the Pressure Implicit Splitting Operator (PISO) to rectify the pressurevelocity system (for details, the interested reader is referred to the work by Jasak, 1996).

The Navier-Stokes equations can be written as

$$
\begin{aligned}
& \nabla \cdot \mathbf{U}=0 \\
& \begin{array}{l}
\frac{\partial \rho \mathbf{U}}{\partial t}+\nabla \cdot(\rho \mathbf{U}) \mathbf{U}-\nabla \cdot\left(\mu_{e f f} \nabla \mathbf{U}\right) \\
\quad=-\nabla p^{*}-(\mathbf{g} \cdot \mathbf{x}) \nabla \rho+\nabla \mathbf{U} \cdot \nabla \mu_{e f f}+\mathbf{F}_{\sigma}
\end{array}
\end{aligned}
$$

where $\rho$ is the fluid density, $\mu_{\text {eff }}$ is the dynamic viscosity, $\mathbf{U}$ is the fluid velocity vector, $\mathbf{x}$ is the position vector, and $\mathbf{g}$ is the acceleration due to gravity pointing downward. The modified pressure $p^{*}$ is defined as

$p^{*}=p-\rho \mathbf{g} \cdot \mathbf{x}$

which has advantages of specifying the pressure on the boundary and stabilizing numerical simulations (Rusche, 2002).

The last term $\mathbf{F}_{\sigma}$ in Eq. 2 is the effect of surface tension, defined by

$\mathbf{F}_{\sigma}=\sigma \kappa \nabla \alpha$
$\kappa=\nabla \cdot \frac{\mathbf{n}}{|\mathbf{n}|}=\nabla \cdot \frac{\nabla \alpha}{|\nabla \alpha|}$

where $\sigma$ is the surface tension coefficient, which is 0.07 in this study; $\boldsymbol{\kappa}$ is the surface curvature; $\mathbf{n}$ is the unit normal vector on 
the free surface pointing outward; and $\alpha$ is the volume fraction, a scalar function defined as

$\alpha(\mathbf{x}, t)= \begin{cases}0, & \text { air } \\ \in(0,1), & \text { free surface } \\ 1, & \text { water }\end{cases}$

That is to say, the volume fraction function is zero at any cell that is totally filled with air and unity if the cell is fully occupied by water. A value between 0 and 1 indicates that the air-water interface cuts the cell, and a curve cutting the cell can be constructed based on this value to approximate the actual interface.

In the VOF method, the air and water phase calculations are integrated into a unified equation by the volume fraction $\alpha$, which can be written as

$\frac{\partial \alpha}{\partial t}+\alpha \nabla \cdot \mathbf{U}+\alpha(1-\alpha) \nabla \cdot \mathbf{U}_{r}=0$

where $\mathbf{U}_{r}$ is a relative compression velocity, artificially used to prevent numerical diffusion of the interface. A detailed description of the artificial compression term can be found in Weller et al. (1998).

The motions of air and water are calculated at the same time as a mixed flow, and the local density and dynamic viscosity can be determined as

$$
\begin{aligned}
& \rho=\alpha \rho_{w}+(1-\alpha) \rho_{a} \\
& \mu=\alpha \mu_{w}+(1-\alpha) \mu_{a}
\end{aligned}
$$

where subscripts $w$ and $a$ refer to water and air, respectively.

\section{Numerical Wave Tank}

To generate and absorb waves, special boundary conditions should be applied for the inlet and outlet of a numerical wave tank. According to the extant literature, the Dirichlet boundary condition used at the location of the wavemaker with specific water particle velocities and surface elevation is proved to be accurate and efficient in constructing a numerical wave tank (Wei and Kirby, 1995; Jacobsen et al., 2012; Higuera et al., 2013). The values of water particle velocities and surface elevation should be specified by the target wave theory and the expected wave parameters. On the basis of this method, the interFoam solver was further extended to the waves2Foam by Jacobsen et al. (2012) with a fully nonlinear wave generation and absorption program. Furthermore, the waves are computed with fully nonlinear potential wave solver OceanWave3D (Engsig-Karup, 2009), and these are coupled to waves2Foam through the interface described by Paulsen et al. (2014a). By these means, we can easily simulate the target wave in a relatively small computational domain and not have to worry about wave reflections. This numerical wave tank technique is used here because of its suitability in coupling specific wave conditions.

The relaxation technique is applied to remove the reflected or internally generated waves. Relaxation zones are used at both ends of the numerical wave tank-the inlet zone and the outlet zone. Within the relaxation zone, the water particle velocity $\mathbf{U}$ and surface elevation $\eta$ are relaxed in each time step by the following function:

$\Phi(x, t)=\xi(\sigma) \Phi(x, t)_{\text {target }}+(1-\xi(\sigma)) \Phi(x, t)_{\text {computed }}$

where $\Phi$ represents either $\mathbf{U}$ or $\eta ; \Phi(x, t)_{\text {target }}$ is the value given by target wave theory parameters; $\Phi(x, t)_{\text {computed }}$ is the value com- puted by Eqs. 1, 2, and 7; and $\xi(\sigma)$ is the relaxation factor, defined as

$$
\xi(\sigma)=\left\{\begin{array}{l}
1-\frac{\exp \left(\sigma^{3.5}\right)-1}{\exp (1)-1}, \\
\sigma \in[0,1], \text { for the inlet relaxation zone } \\
\frac{\exp \left(\sigma^{3.5}\right)-1}{\exp (1)-1}, \\
\sigma \in[0,1], \text { for the outlet relaxation zone }
\end{array}\right.
$$

where $\sigma$ is the normalized horizontal coordinate along the relaxation zone. In general, the relaxation zones should be long enough (i.e., exceeding the reflecting wave length) to ensure that there is no wave re-reflection.

\section{Force on Structures}

By establishing the numerical wave tank and solving the coupled velocity-pressure equation, we can compute the pressure field. Once the pressure distribution on a structure is obtained, one can readily calculate the wave force on the structure by integrating the pressure over the wetted surface of the structure, namely (Paulsen et al., 2014b)

$\mathbf{F}(t)=-\int_{\Omega} p(x, y, z, t) d \mathbf{n}$

where $\mathbf{F}(t)$ is the wave force, $\mathbf{n}$ is the outward normal vector of the structure surface, and $\Omega$ is the wetted area of the structure. It should be noted that interFoam is a two-phase flow solver, but the wave forces are computed for the water phase only.

\section{Validation}

The accuracy and feasibility of the numerical wave tank play an important role in simulations. A large amount of validation has been done by Jacobsen et al. (2012) and Paulsen et al. (2014b). In this study, a further validation of wave elevation and wave force on a cylinder is carried out. The numerical results are compared with theoretical predictions of the stream function theory and the Morison equation.

A numerical experiment is set up with the following parameters to account for nonlinear effect. This experiment is of a fully nonlinear wave of height $H=5.8 \mathrm{~m}$, period $T=7.8 \mathrm{~s}$, water depth
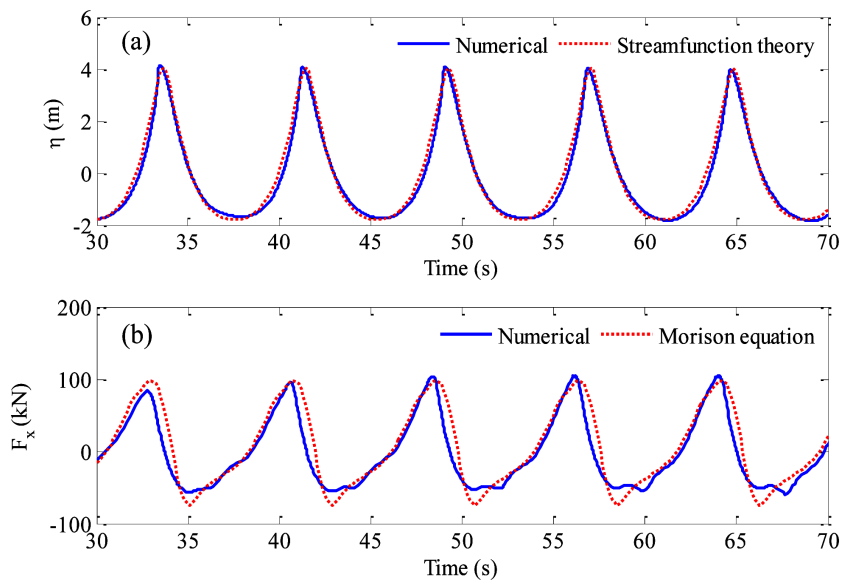

Fig. 2 Comparison between numerical results and theoretical predictions for a fully nonlinear wave: (a) the free-surface elevation and (b) the horizontal force on a cylinder 
$h=11 \mathrm{~m}$, and cylinder diameter $D=1.7 \mathrm{~m}$. The load coefficients of the Morison equation are $C_{D}=1.2$ and $C_{M}=1.8$, as referenced in Sarpkaya and Isaacson (1981). Good agreements are found in Fig. 2, although there is a small discrepancy between the numerical result and the Morison equation in horizontal force. That is due to the nonlinear effect and the water reflow around the cylinder after the crest has passed. It can be seen in Fig. $2 b$ that the maximum negative force of the numerical result is smaller than that of the Morison equation. This phenomenon was also found by Paulsen et al. (2014b), who defined it as the secondary load cycle.

For the practical structure in the East China Sea, the bottom elevation of the cap is $-0.3 \mathrm{~m}$, which is very close to the still water level. The free water surface can hit the bottom of the cap. This impact phenomenon plays an important role in the total loads of this type of structure. Hence, it is necessary to make a validation of the impact force between the free water surface and a fixed structure, which is carried out here by a classical dam break problem.

The typical setting of the dam break problem is identical to the experiment described by Kleefsman et al. (2005). The scale of the tank is $3.22 \times 1 \times 1 \mathrm{~m}^{3}$ with an open roof. In the tank, a fixed box with a scale of $0.16 \times 0.4 \times 0.16 \mathrm{~m}^{3}$ is placed on the bottom, as shown in Fig. 3a. The distance between the left wall of the tank and the left side of the box is $0.67 \mathrm{~m}$. There are two pressure sensors set on the box: P1 $(0.83 \mathrm{~m},-0.026 \mathrm{~m}, 0.025 \mathrm{~m})$ on the right side and P2 $(0.806 \mathrm{~m}, 0.026 \mathrm{~m}, 0.16 \mathrm{~m})$ on the top of the box; see Fig. 3b. In the right part of the tank, from $x=2 \mathrm{~m}$, water at a height of $0.55 \mathrm{~m}$ is initially kept still. When the simulation starts, the water flows down toward the left wall and impacts the box. A fine grid of $232 \times 110 \times 90$ cells gradually refined from the open area toward the wall is used in this case. The time histories of the pressure measured at P1 and P2 are presented in Fig. 4. For $\mathrm{P} 1$, good agreement is seen. In particular, the magnitude of the impact pressure is almost the same. For P2, the impact pressure is overestimated by simulation and seems to violently change when compared with the experimental measurement. It should be noted

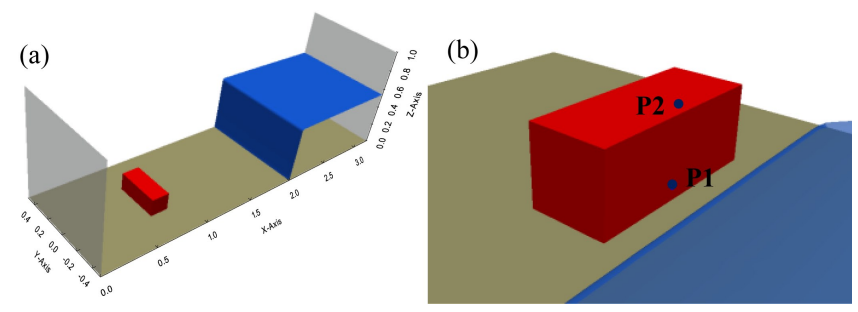

Fig. 3 The dam break problem with a box: (a) initial configuration and (b) positions for pressure measurement
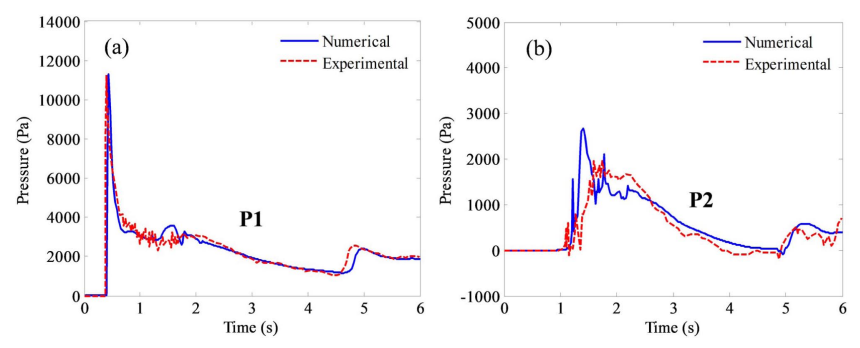

Fig. 4 Time histories of the pressures at (a) P1 and (b) P2: a comparison between the numerical results (solid line) and the laboratory experiment (dashed line; Kleefsman et al., 2005) shows good agreement. that this numerical solver is an incompressible flow model for both water and air. The phenomenon of these violent changes of pressure is probably a result of the compressibility of air when the uplifted water falls down and impacts the top of the box with air cavities. However, these big air cavities rarely appear in the following investigation of the present new structure. Taking this into account, we deem that the impact force between the free water surface and a high-rise-pile-cap structure can be simulated accurately by this numerical model.

\section{PARAMETER SETTINGS AND GRID CONVERGENCE}

\section{Wave Parameters and Structure Settings}

The Donghai Bridge Wind Farm is located in a shallow water area with a depth of $11 \mathrm{~m}$. In the present study, we choose the highest designed wave height of $H_{1 \%}$-namely, the wave height with cumulative frequency of $1 \%$. Its return period is 50 years. The wave length is $75 \mathrm{~m}$, the wave height is $5.8 \mathrm{~m}$, and the period is $7.8 \mathrm{~s}$, which is close to the limit of wave breaking. This nonlinear wave is achieved by stream function theory (Fenton, 1988) with 20-order truncation. These are exactly the in-situ hydrodynamic environments for the wind farm.

The simplified physical domain and the structure supporting offshore wind turbines are sketched in Fig. 5a. The computational model parameters are consistent with the actual structure. Specifically, the structure consists of a circular surface piercing cap $14 \mathrm{~m}$ in diameter and eight supporting piles $1.7 \mathrm{~m}$ in diameter with a slope ratio of 5.5 to 1 . A high vertical tower $4.5 \mathrm{~m}$ in diameter is mounted to the platform. The cap with a draught of $0.3 \mathrm{~m}$ in still water consists of two parts. The lower part is a cylinder $3 \mathrm{~m}$ in height, and the upper one is a frustum of a cone with a half-meter height. The inclined piles are evenly mounted along a circle with a radius of $5 \mathrm{~m}$ on the bottom of the cap. To facilitate the subsequent analysis, we identify piles 1 to 5 as shown in Fig. 5 b.

\section{Grid Convergence}

It is very necessary to make sure that the simulation results are independent of grid resolution. The grid is shown in Fig. 6 and is gradually refined from the open offshore boundary toward the structure. Slip wall conditions are applied at the seabed, lateral boundaries, and structure wall. This boundary condition increases the computational efficiency, because no numerical resolution of the structural boundary layer is needed. More importantly, the wave forces are inertia dominated for the present computations. Therefore, neglecting the structure wall boundary layer can be a reasonable approximation (Paulsen et al., 2014b).

The grid convergence is investigated by three different mesh configurations shown in Table 1 , where $\Delta s$ is the thickness of the first grid layer from the wall. The near-field grid becomes finer and finer going from mesh 1 to mesh 3 to account for the complicated interactions between the waves and the structure. The total horizontal forces on the high-rise pile cap structure are calculated using each of these mesh configurations and are presented as a

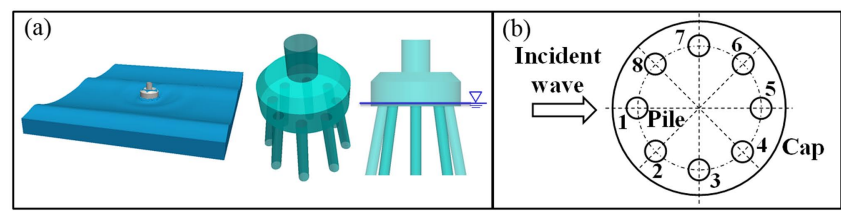

Fig. 5 The simplified physical domain with a high-rise pile cap structure in it and (b) the locations relative to the incident wave of the piles on which the numerical pressure is measured 


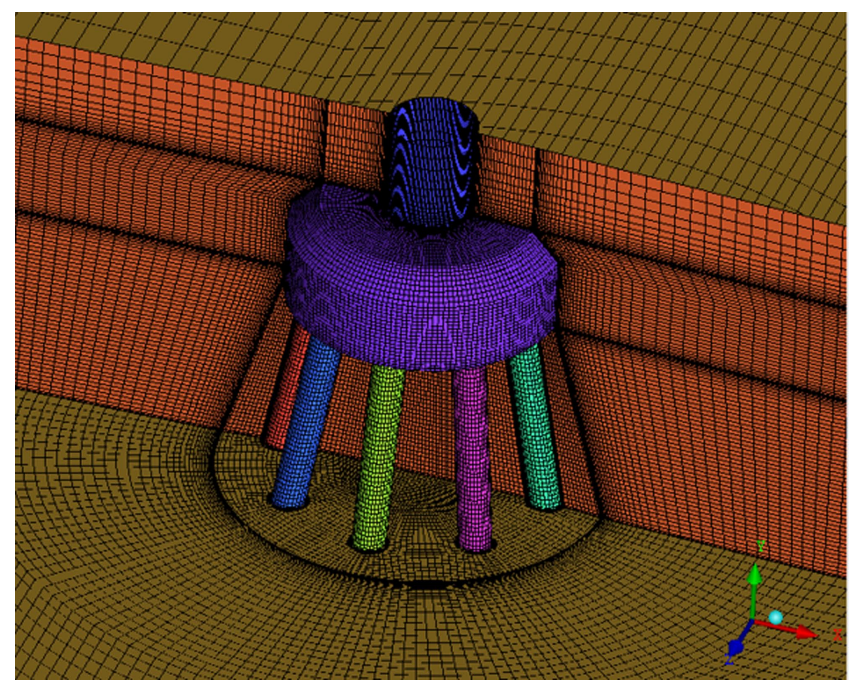

Fig. 6 Numerical grid for the high-rise pile cap structure

\begin{tabular}{lccc}
\hline & Mesh 1 & Mesh 2 & Mesh 3 \\
\hline Piles cells & $1,224 \times 8$ & $1,800 \times 8$ & $2,880 \times 8$ \\
Cap cells & 14,716 & 18,012 & 31,172 \\
Near-wall grid $\Delta s(\mathrm{~m})$ & $6.25 \mathrm{e}-5$ & $5 \mathrm{e}-5$ & $4 \mathrm{e}-5$ \\
Near-field cells & 480,664 & 909,328 & $1,740,680$ \\
Total cells, $x$ direction & 120 & 132 & 152 \\
Total cells, $y$ direction & 82 & 86 & 106 \\
Total cells, $z$ direction & 72 & 95 & 111 \\
Total number of cells & $1,085,176$ & $1,820,188$ & $3,102,428$ \\
\hline
\end{tabular}

Table 1 Mesh configurations used for grid convergence examination
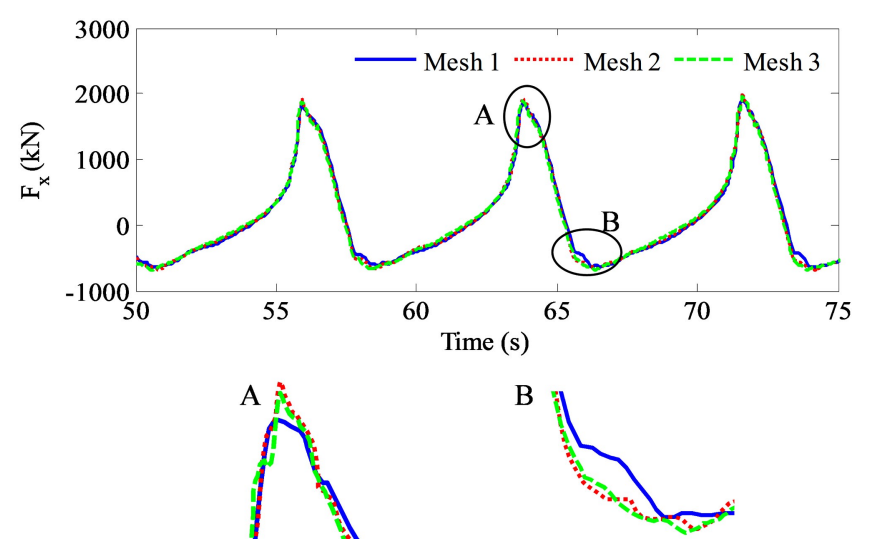

Fig. 7 Time histories of the horizontal force on the entire structure for different meshes

function of time in Fig. 7. It is found that the horizontal forces computed using three different meshes are alike, whereas meshes 2 and 3 have better descriptions for the peak and valley values. With a trade-off consideration of precision and computational efficiency, the mesh 2 configuration is the most appropriate for this numerical experiment.

\section{RESULTS AND DISCUSSION}

In general, a large cap in the wave field has a significant influence on water motion, especially for the surface piercing struc- tures such as those in the present investigation. To reveal the effect of the interaction between the cap and the piles on their wave loads, we consider three types of settings: piles only (case A), cap only (case B), and the whole supporting structure (case C).

\section{Wave Loads on Piles with Cap}

When the cap exists, the wave loads on piles are influenced not only by the diffraction caused by the cap but also by the impact of the water surface on the cap bottom seriously, which can rarely be predicted by traditional methods, such as the Morison equation, the diffraction theory, or other simple models. Fortunately, this special load resulting from the high pressure generated by the impact can be obtained by integrating the pressure over the wetted area of the piles using the numerical method described above.

Time histories of the horizontal force on the piles for cases A and $\mathrm{C}$ are shown in Fig. 8. In case $\mathrm{A}$, the maximum horizontal force is about $100 \mathrm{kN}$. There are no obvious differences in the maximum horizontal force for each pile except for the wave-phase differences among the piles, which can be anticipated in advance (see Fig. 8a). The influence of the shadowing effect of pile groups on the maximal wave horizontal force is not significant. However, Fig. 8b shows that in case $\mathrm{C}$ the horizontal force becomes quite different at the peak of its duration curve, and the maximum horizontal force can be much greater than that of case A. The detail of the third peak around $t=48 \mathrm{~s}$ is shown in Fig. 8c. The figure shows that the horizontal forces of piles 1 and 2 have a sharp decrease at the moment featuring a volcano-like structure, whereas the horizontal forces of piles 3-5 increase dramatically.

To explain this phenomenon, the pressure fields at three moments on a horizontal plane located at $3 \mathrm{~m}$ below the still water level are presented in Fig. 9. The snapshots at $t=47.5 \mathrm{~s}$ show
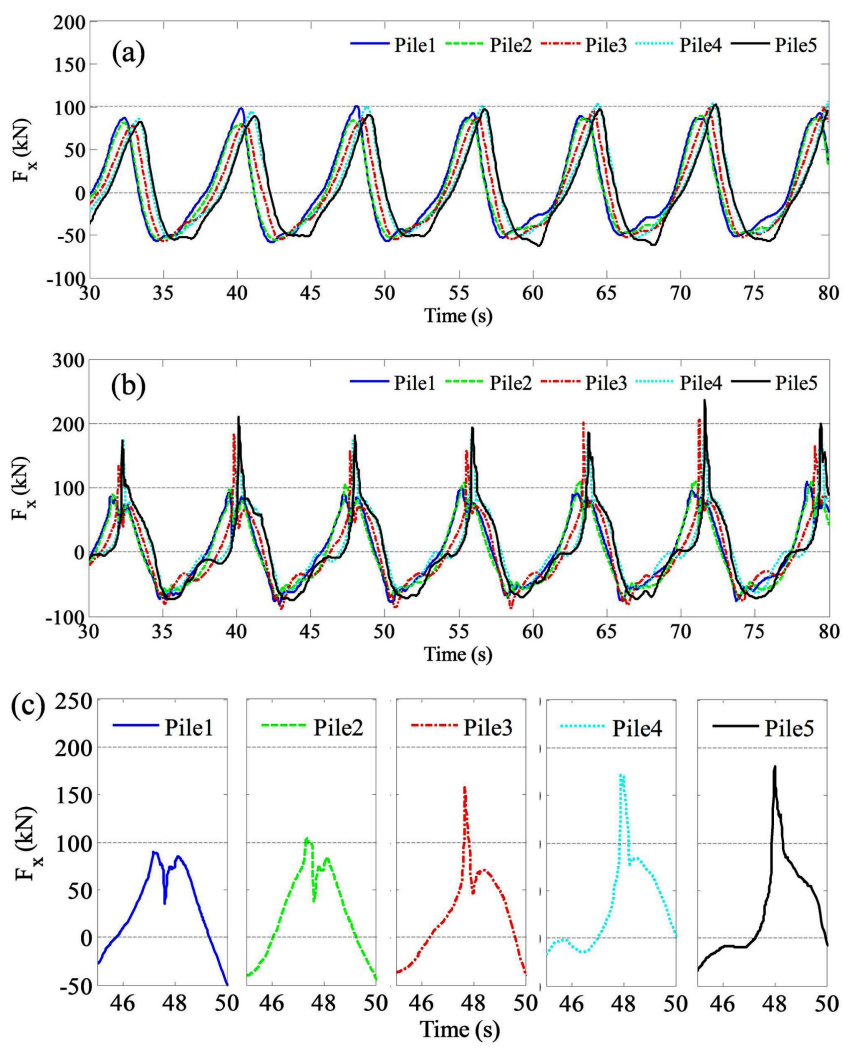

Fig. 8 Time histories of horizontal force on each pile: (a) piles only (case A), (b) the whole supporting structure (case C), and (c) a magnified view of the third peak around $t=48 \mathrm{~s}$ for case $\mathrm{C}$ 

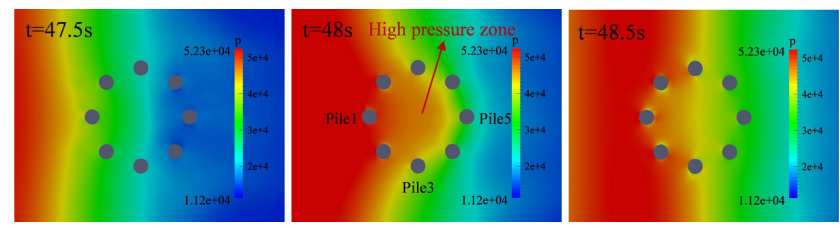

Fig. 9 The pressure field on the horizontal plane $3 \mathrm{~m}$ below the still water level at three moments during impact; the colors show pressure in pascals.

the pressure field just before the wave impacts the cap bottom. At $t=48 \mathrm{~s}$, the wave surface is impacting on it, and at $t=48.5 \mathrm{~s}$, the cap bottom is submerged. We can easily find that the pressure variances are coincident with the wave direction, either before impact $(t=47.5 \mathrm{~s})$ or after impact $(t=48.5 \mathrm{~s})$. In other words, the pressures are almost the same within a certain wave phase. However, when the impact takes place $(t=48 \mathrm{~s})$, there exists an instantaneous high pressure zone below the cap. This means that the vertical impact to the cap bottom causes an increase in fluid pressure, which generates an additional horizontal pressure gradient. Thus, integrating pressure over the pile surface leads to an instantaneous additional load with different directions for different piles. Hence, the upstream piles 1 and 2 have additional negative horizontal forces, whereas the downstream piles 3-5 have additional positive ones. The maximum horizontal force generally occurs on pile 5 .

Figure 10 presents the vertical distribution of pressure increase $\Delta p=p_{C}-p_{A}$ in water phase along the central line of the structure at $t=48 \mathrm{~s}$, where $p_{A}$ and $p_{C}$ are the pressures in case $\mathrm{A}$ and case $\mathrm{C}$, respectively. As indicated in Fig. 10, there is a pressure increase along the whole central line. In addition, the nearer to the cap bottom, the more the pressure increases. This pressure increase grows fast above the position higher than $-6 \mathrm{~m}$.

The free surface evolutions and the water particle velocities during the impact are presented in Fig. 11 to further confirm the reason for pressure increase. The figure indicates that water particles impact on the cap bottom with the vertical velocities exceeding $2 \mathrm{~m} / \mathrm{s}$, and then the vertical velocities suddenly vanish. Consequently, a water flow with horizontal velocity exceeding $4 \mathrm{~m} / \mathrm{s}$ emerges near the cap bottom and hits on the downstream pile 5 . As a result, except for the increasing load caused by the high pres-

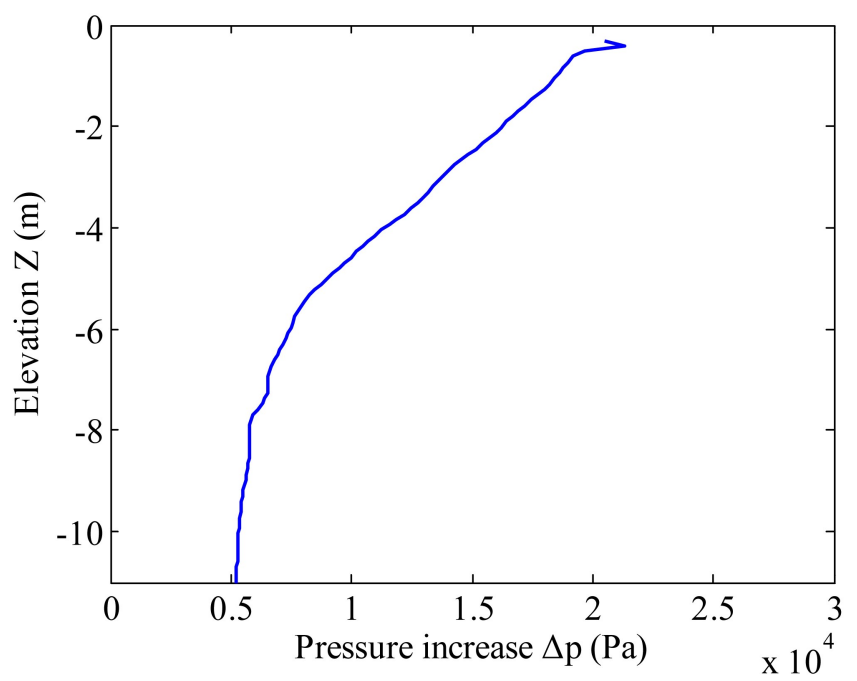

Fig. 10 Vertical distribution of an additional pressure increase $\Delta p$ in water phase along the central line of the structure during impact
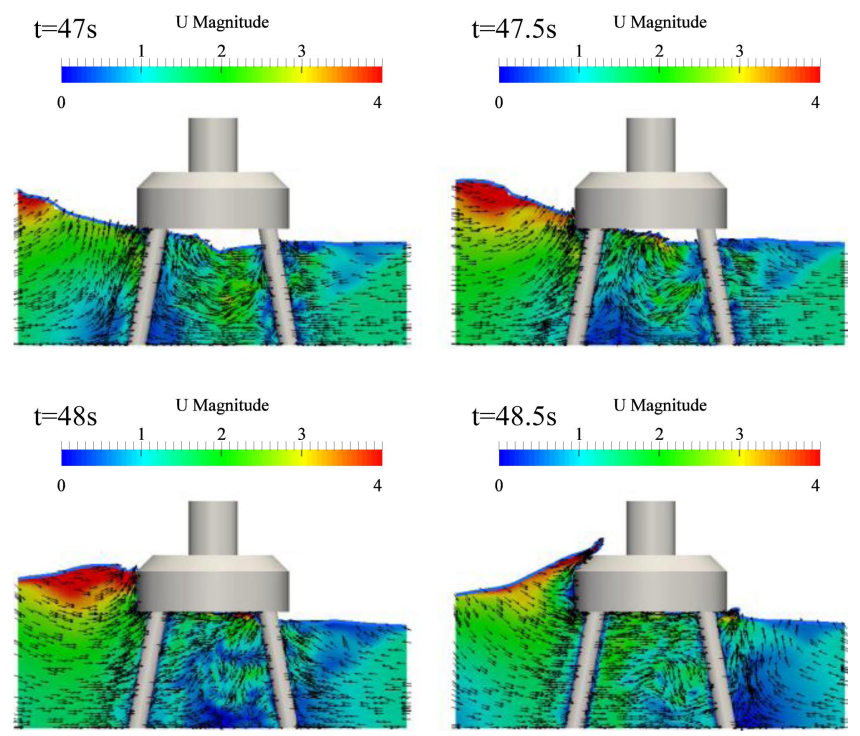

Fig. 11 Free surface evolutions and water particle velocities during the impact

sure zone discussed above, the downstream piles suffer an extra load by the direct impact of the high-speed horizontal water flow. Figure 12 shows the difference of surface pressure distributions on all eight piles between cases A and C, where $\rho g h$ represents the hydrostatic pressure and $p-\rho g h$ is the dynamic pressure. The pressure along the piles in case $\mathrm{C}$ is generally greater than that of case A, especially for the top parts of the piles. Moreover, there are some obvious higher pressure areas on the downstream piles. These high pressure areas close to the cap bottom are likely caused by the water flow impact. Figure 13 presents the pressure distribution along the headmost generatrix against the incident flow of pile 5 at $t=48 \mathrm{~s}$. We can see a high pressure increase at the top of the pile, and the maximum value reaches to $80 \mathrm{kPa}$. This instantaneous impact load not only puts forward the requirement for the local bearing capacity of the pile-cap junction but also affects the fatigue damage of the structure because of its periodicity.

As mentioned above, piles 1 and 2 have additional negative horizontal forces when the cap exists, whereas piles 3-5 have additional positive ones. Another concerned question is how the total force will change. The time series of the total horizontal force and moment (to the intersection of the central line of the

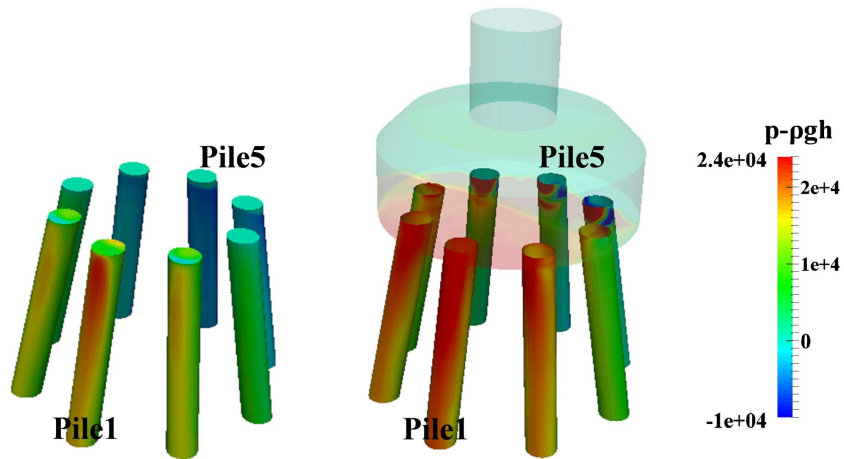

(a) Piles only

(b) Piles with cap

Fig. 12 Pressure distributions on the piles' surface at $t=48 \mathrm{~s}$ : (a) piles only (case A) and (b) the whole supporting structure (case C) 


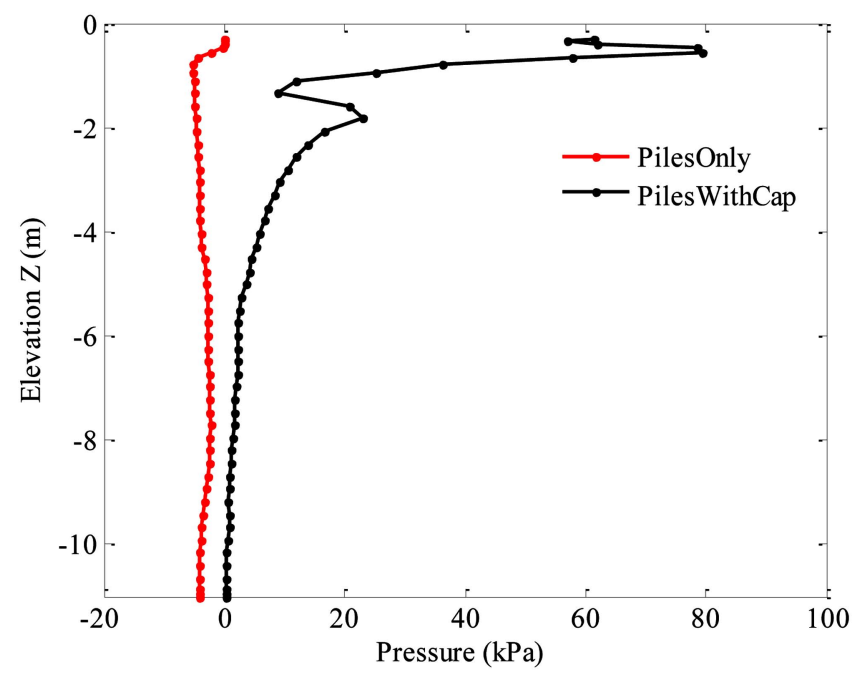

Fig. 13 Pressure distributions along the most headmost generatrix against the incident flow of pile 5 at $t=48 \mathrm{~s}$
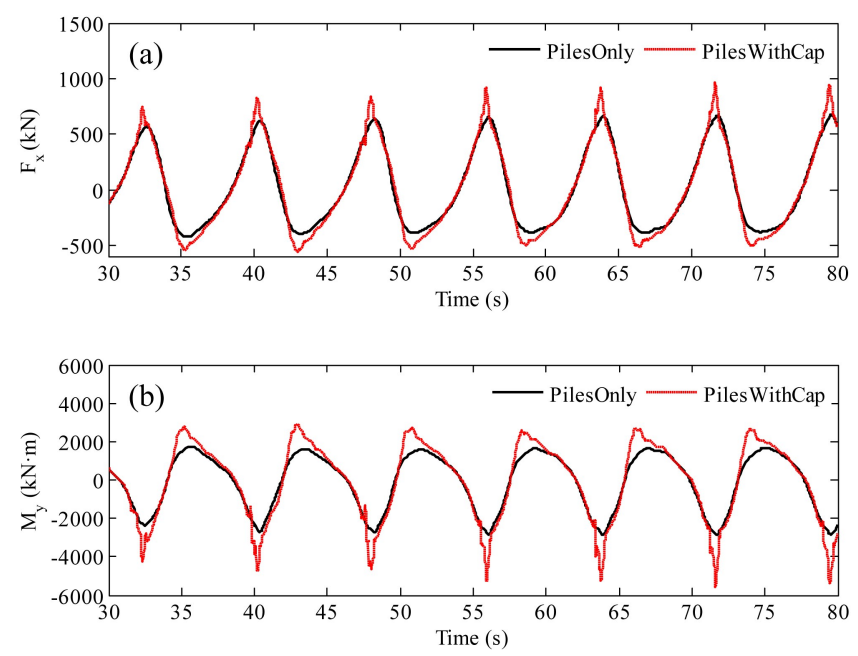

Fig. 14 Comparison of time histories of the total wave load of the piles between case A and case C: (a) horizontal force of the piles and (b) moment of the piles

whole structure and the sea bed) of all eight piles are given in Fig. 14. Comparing cases $\mathrm{A}$ and $\mathrm{C}$, the total horizontal force of all the piles increases by about $30 \%$ when the cap exits, whereas the total moment is doubled.

\section{Wave Loads on the Cap with Piles}

Although the piles also have an obvious influence on water motion, the wave loads on the cap are not as sensitive as those on the piles that have been discussed above. The time series of the horizontal force, the vertical force, and the moment (to the intersection of the central line of the whole structure and the sea bed) of the cap are presented in Fig. 15. Comparing cases B and C, the horizontal force increases by about $15 \%$ when the piles exist, and the total moment increases by about $5 \%$, but the vertical force decreases by about $7 \%$. The increase of the horizontal force is mainly due to the blockage of the piles to wave motion, which makes the wave surface uplift slightly, and a larger water body push on the cap. When there are piles below the cap, the wetted area of the cap bottom decreases by about $6 \%$. Hence, the decrease of the vertical force is probably due to the discount of the impact area.
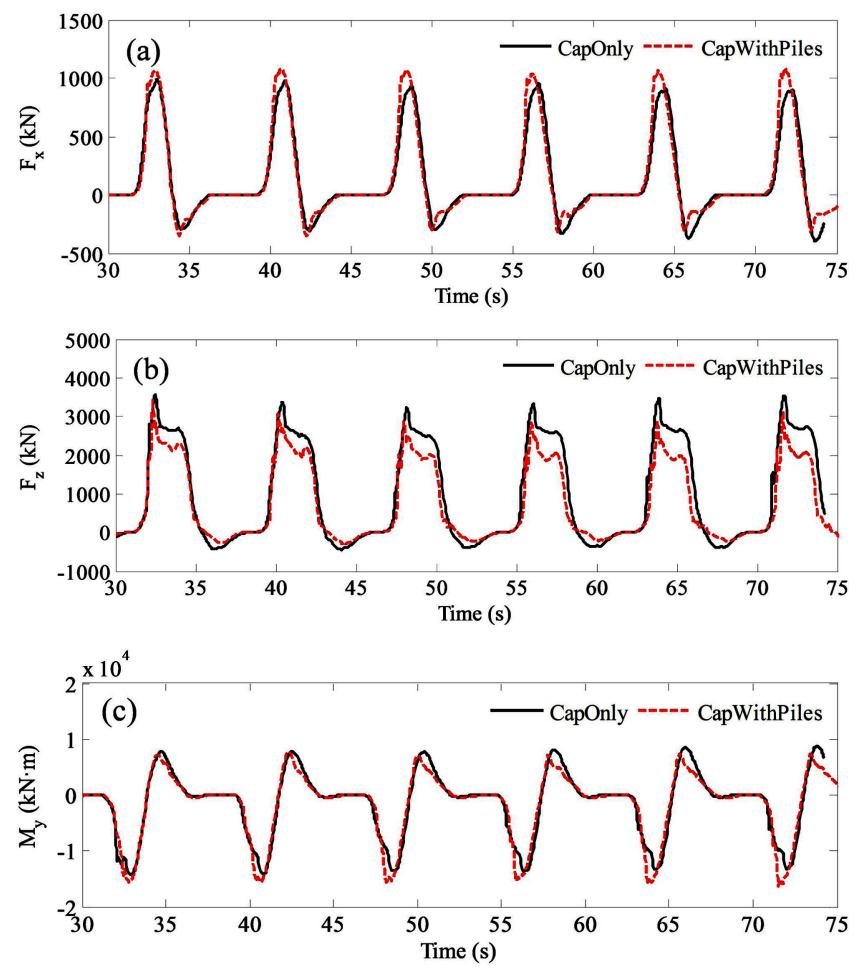

Fig. 15 Comparison of time histories of the total wave load between case B and case C: (a) horizontal force of the cap, (b) vertical force of the cap, and (c) moment of the cap

\section{Wave Loads of the Piles and the Cap by Means of Conventional Methods}

Now, we can see that the wave load on the pile group is greatly affected by the impact. This may be significantly beyond the prediction of conventional engineering methods. In engineering, the conventional Morison equation and the diffraction theory often give good estimates of the forces on small-scale or large-scale structures, respectively, for linear waves with no impact. However, when impinging on the cap of the investigated structure takes place, the velocity and pressure fields deviate far away from those described by linear theories, which makes the conventional methods incapable of estimating the forces of both the small-scale piles and large-scale cap of the structure. For example, as shown in Table 2, using the Morison equation to calculate the horizontal force of the piles in case $\mathrm{C}$, the maximum horizontal forces of piles $1,2,3,4$, and 5 are $105.8 \mathrm{kN}, 104.9 \mathrm{kN}, 104 \mathrm{kN}, 104.9 \mathrm{kN}$, and $105.8 \mathrm{kN}$, respectively, which obviously underestimate the maximum horizontal forces of the downstream piles. In addition,

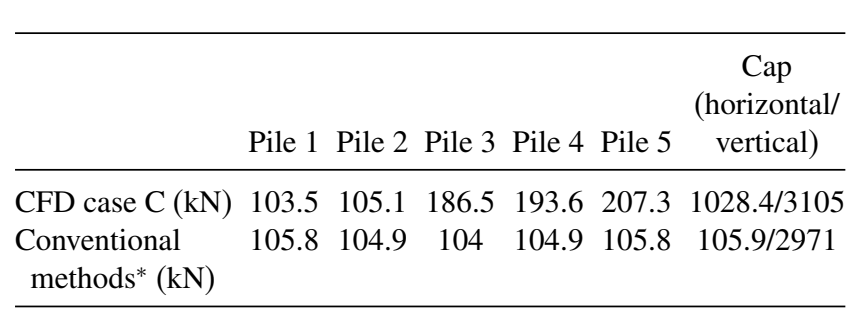

*By "conventional methods," we mean that the wave forces of the cap are calculated by linear diffraction theory, and that the wave forces of the piles are calculated by the Morison equation using the velocity field obtained from the diffraction theory for the cap. Table 2 Comparison of wave load predictions between CFD and the conventional methods 
the results of the linear diffraction theory are $105.9 \mathrm{kN}$ for the horizontal force and $2,971 \mathrm{kN}$ for the vertical force on the cap. The horizontal force is greatly underestimated, which is mainly due to the shallow draught and fully nonlinear effect. A highorder diffraction theory might give a better estimation, but it is too complicated to apply in engineering. The vertical force is also underestimated apparently because the diffraction theory ignores the impact effect. Overall, the wave loads of the present composite structure can hardly be estimated with these conventional approaches. The impact effect between the free surface and the cap should be taken into consideration carefully, especially for the case of impinging on the cap bottom.

\section{CONCLUSIONS}

We carried out a numerical investigation for wave loads of the high-rise pile cap foundation of an offshore wind turbine being used in the Donghai Bridge Wind Farm. To begin with, a fully nonlinear numerical wave tank based on the Navier-Stokes equations is established, where the nonlinear waves are obtained by the 20-order stream function theory. The wave loads and moments are obtained by integrating the pressure over the wetted surface of the structure. We have figured out direct and indirect consequences of the wave impact on the cap bottom, which is of significance and was overlooked in the design of the high-rise pile cap structure.

In the case of piles only, the magnitudes of the horizontal wave force for each pile are almost the same, and the maximum horizontal force is about $100 \mathrm{kN}$. In the case of the whole high-rise pile cap structure, the maximum horizontal force of a single pile can be up to $200 \mathrm{kN}$. A sharp rise toward the maximum load occurs at the moment of free surface impinging on the cap bottom. This impact induces a high pressure zone below the cap. At that moment, the horizontal forces of piles 1 and 2 have a sharp decrease, whereas those of piles 3-5 increase dramatically. The maximum horizontal force generally occurs on pile 5. Furthermore, the total horizontal force of all the piles increases by about $30 \%$ when the cap exits, and the total moment is doubled.

The existence of the piles also affects the wave load of the cap. When piles are considered, the horizontal force increases by about $15 \%$ and the total moment increases by about $5 \%$, but the vertical force decreases by about $7 \%$.

In engineering, the conventional methods, such as the Morison equation and the diffraction theory, often give good estimates of forces for small-scale piles or large-scale caps, respectively, for nonbreaking waves. However, wave loads of the present composite structure can hardly be estimated with these approaches. The wave impact effect should be taken into serious consideration, especially for the impinging on the cap bottom. The new findings of this paper may serve as a fundamental basis to optimize the high-rise pile cap structure for further wind farm constructions.

\section{ACKNOWLEDGEMENTS}

This work was supported by the National Natural Science Foundation of China (Grants 11232012, 11572332, and 11172307), National Key R\&D Program of China (2017YFC1404202, 2014CB046204), and the Strategic Priority Research Program of the Chinese Academy of Sciences (Grant XDB22040203).

\section{REFERENCES}

Azadbakht, M, and Yim, SC (2016). "Effect of Trapped Air on Wave Forces on Coastal Bridge Superstructures," J Ocean Eng Mar Energy, 2(2), 139-158.

https://doi.org/10.1007/s40722-016-0043-9.
Bredmose, H, and Jacobsen, NG (2011). "Vertical Wave Impacts on Offshore Wind Turbine Inspection Platforms," Proc 30th Int Conf Ocean Offshore Arct Eng, Rotterdam, Netherlands, ASME, 5, 645-654. https://doi.org/10.1115/OMAE2011-49785.

Chang, KT, Jeng, DS, Zhang, JS, and Zhang, Y (2014). "Soil Response around Donghai Offshore Wind Turbine Foundation, China," Proc Inst Civ Eng Energy, 167(1), 20-31. https://doi.org/10.1680/ener.12.00017.

Chen, L, Zhou, JF, Li, J, Lin, YF, and Li, JY (2016). "Monitoring the New Fixed Offshore Wind Turbine Foundation in the East China Sea," J Ocean Wind Energy, ISOPE, 3(1), 10-15. https://doi.org/10.17736/jowe.2016.jcr38.

Chen, LF, Zang, J, Hillis, AJ, Morgan, GCJ, and Plummer, AR (2014). "Numerical Investigation of Wave-structure Interaction Using OpenFOAM," Ocean Eng, 88, 91-109.

https://doi.org/10.1016/j.oceaneng.2014.06.003.

Cuomo, G, Shimosako, KI, and Takahashi, S (2009). "Wave-indeck Loads on Coastal Bridges and the Role of Air," Coastal Eng, 56(8), 793-809. https://doi.org/10.1016/j.coastaleng.2009.01.005.

Engsig-Karup, AP, Bingham, HB, and Lindberg, O (2009). "An Efficient Flexible-order Model for 3D Nonlinear Water Waves," J Comput Phys, 228(6), 2100-2118. https://doi.org/10.1016/j.jcp.2008.11.028.

Fenton, JD (1988). "The Numerical Solution of Steady Water Wave Problems," Comput Geosci, 14(3), 357-368. https://doi.org/10.1016/0098-3004(88)90066-0.

Hayatdavoodi, M, Seiffert, B, and Ertekin, RC (2014). "Experiments and Computations of Solitary-wave Forces on a Coastalbridge Deck. Part II: Deck with Girders," Coastal Eng, 88, 210-228. https://doi.org/10.1016/j.coastaleng.2014.02.007.

Higuera, P, Lara, JL, and Losada, IJ (2013). "Realistic Wave Generation and Active Wave Absorption for Navier-Stokes Models: Application to OpenFOAM," Coastal Eng, 71, 102-118. https://doi.org/10.1016/j.coastaleng.2012.07.002.

Higuera, P, Lara, JL, and Losada, IJ (2014). "Three-dimensional Interaction of Waves and Porous Coastal Structures Using OpenFOAM®. Part I: Formulation and Validation," Coastal Eng, 83, 243-258. https://doi.org/10.1016/j.coastaleng.2013.08.010.

Hirt, CW, and Nichols, BD (1981). "Volume of Fluid (VOF) Method for the Dynamics of Free Boundaries," J Comput Phys, 39(1), 201-225. https://doi.org/10.1016/0021-9991(81)90145-5.

$\mathrm{Hu}, \mathrm{ZZ}$, Greaves, D, and Raby, A (2016). "Numerical Wave Tank Study of Extreme Waves and Wave-structure Interaction Using OpenFoam ${ }^{\circledR}, "$ Ocean Eng, 126, 329-342. https://doi.org/10.1016/j.oceaneng.2016.09.017.

Iwanowski, B, Gladsø, R, and Lefranc, M (2009). "Wave-indeck Load on a Jacket Platform, CFD-derived Pressures and Non-linear Structural Response," Proc 28th Int Conf Ocean Offshore Arct Eng, Honolulu, HI, USA, ASME, 1, 31-40. https://doi.org/10.1115/OMAE2009-79053.

Jacobsen, NG, Fuhrman, DR, and Fredsøe, J (2012). "A Wave Generation Toolbox for the Open-source CFD Library: OpenFoam®," Intl J Numer Methods Fluids, 70(9), 1073-1088. https://doi.org/10.1002/fld.2726.

Jasak, H (1996). Error Analysis and Estimation for the Finite Volume Method with Applications to Fluid Flows, PhD thesis, University of London, London, UK. 
Kamath, A, Alagan, CM, Bihs, H, and Arntsen, ØA (2015). "Evaluating Wave Forces on Groups of Three and Nine Cylinders Using a 3D Numerical Wave Tank," Eng Appl Comput Fluid Mech, 9(1), 343-354. https://doi.org/10.1080/19942060.2015.1031318.

Kleefsman, KMT, Fekken, G, Veldman, AEP, Iwanowski, B, and Buchner, B (2005). "A Volume-of-fluid Based Simulation Method for Wave Impact Problems," J Comput Phys, 206(1), 363-393. https://doi.org/10.1016/j.jcp.2004.12.007.

Li, Y, and Lin, M (2012). "Regular and Irregular Wave Impacts on Floating Body," Ocean Eng, 42, 93-101. https://doi.org/10.1016/j.oceaneng.2012.01.019.

Lin, YF, Li, JY, Shen, D, and Song, C (2007). "Structure Characteristics and Design Technique Keys of Turbine Foundation in Shanghai Donghaid-Bridge Offshore Wind Farm," Shanghai Electr Power, 2, 153-157. (In Chinese)

Lin, Z, et al. (2017). "Investigation of Nonlinear Wave-induced Seabed Response Around Mono-pile Foundation," Coastal Eng, 121, 197-211. https://doi.org/10.1016/j.coastaleng.2017.01.002.

Lu, ZM (2010). "Study on Key Technologies Employed in Planning and Construction of Shanghai Donghai Bridge Offshore Wind Farm," Eng Sci, 12(11), 19-24. (In Chinese)

Morgan, GCJ, et al. (2010). "Using the RasInterFoam CFD Model for Wave Transformation and Coastal Modeling," Coastal Eng Proc, 1(32), 23-31. https://doi.org/10.9753/icce.v32.waves.23.

Paulsen, BT, Bredmose, H, and Bingham, HB (2014a). "An Efficient Domain Decomposition Strategy for Wave Loads on Surface Piercing Circular Cylinders," Coastal Eng, 86, 57-76. https://doi.org/10.1016/j.coastaleng.2014.01.006.

Paulsen, BT, Bredmose, H, Bingham, HB, and Jacobsen, NG (2014b). "Forcing of a Bottom-mounted Circular Cylinder by
Steep Regular Water Waves at Finite Depth," J Fluid Mech, 755, 1-34. https://doi.org/10.1017/jfm.2014.386.

Rusche, H (2002). Computational Fluid Dynamics of Dispersed Two-Phase Flows at High Phase Fractions, PhD thesis, University of London, London, UK.

Sarpkaya, T, and Isaacson, M (1981). Mechanics of Wave Forces on Offshore Structures, Van Nostrand Reinhold, 651 pp.

Schellin, TE, Perić, M, and El Moctar, O (2011). "Wave-in-deck Load Analysis for a Jack-up Platform," J Offshore Mech Arct Eng, 133(2), 021303. https://doi.org/10.1115/1.4002047.

Seiffert, B, Hayatdavoodi, M, and Ertekin, RC (2014). "Experiments and Computations of Solitary-wave Forces on a Coastalbridge Deck. Part I: Flat Plate," Coastal Eng, 88, 194-209. https://doi.org/10.1016/j.coastaleng.2014.01.005.

Seiffert, BR, Ertekin, RC, and Robertson, IN (2015). "Wave Loads on a Coastal Bridge Deck and the Role of Entrapped Air," Appl Ocean Res, 53, 91-106.

https://doi.org/10.1016/j.apor.2015.07.010.

Stansberg, CT, Baarholm, R, Kristiansen, T, Hansen, EWM, and Rortveit, G (2005). "Extreme Wave Amplification and Impact Loads on Offshore Structures," Proc Offshore Technol Conf, Houston, TX, USA, OTC-17487-MS. https://doi.org/10.4043/17487-MS.

Wei, G, and Kirby, JT (1995). “Time-dependent Numerical Code for Extended Boussinesq Equations," J Waterw Port Coastal Ocean Eng, 121(5), 251-261. https://doi.org/10.1061/(ASCE)0733-950X(1995)121:5(251).

Weller, HG, Tabor, G, Jasak, H, and Fureby, C (1998). "A Tensorial Approach to Computational Continuum Mechanics Using Object-oriented Techniques," Comput Phys, 12(6), 620-631. https://doi.org/10.1063/1.168744. 